\section{Finasteride is effective for the treatment of central serous chorioretinopathy}

\begin{abstract}
Purpose To evaluate the safety and efficacy of finasteride treatment in patients with central serous chorioretinopathy (CSC). Methods Retrospective review of 29 eyes of 23 patients who were treated with finasteride for CSC. Previous medical and ocular history, steroid use, length of finasteride treatment, additional treatments for CSC, visual acuity (VA), central macular thickness (CMT), and presence of subretinal fluid (SRF) throughout the follow-up period, and the occurrence of any complications were recorded.

Results Initial VA was $0.29 \pm 0.31 \log$ MAR, and a trend towards improved VA was noted after 3 months $(0.25 \pm 0.36 \log$ MAR; $P=0.07)$. VA was significantly improved at the final follow-up $(0.23 \pm 0.27 \log$ MAR; $P=0.024)$. Initial CMT was $354 \pm 160 \mu \mathrm{m}$, and was significantly reduced after 1 month of treatment ( $284 \pm 77 \mu \mathrm{m} ; P=0.002)$ and this was maintained to the end of follow-up

$(247 \pm 85 \mu \mathrm{m} ; P=0.001)$. A significant reduction in SRF presence was found at all time points, with an overall $75.9 \%$ rate of complete resolution. Following discontinuation, SRF recurrence was noted in $37.5 \%$ of cases. No adverse events were recorded.

Conclusions Finasteride is a safe and effective treatment for CSC. It may be a possible new option for the initial management of patient with CSC, and a suggested treatment approach is presented. Eye (2016) 30, 850-856; doi:10.1038/ eye.2016.53; published online 8 April 2016
\end{abstract}

Department of Ophthalmology \& Vision Science, University of California Davis Eye Center, Sacramento, CA, USA

Correspondence: E Moisseiev, Department of Ophthalmology \& Vision Science, University of California Davis Eye Center, 4860 Y Street, Suite 2400, Sacramento, CA 95817, USA

Tel: +1 916734 6544; Fax: +1 9167346197 .

E-mail: elad_moi@ netvision.net.il

${ }^{1}$ These authors contributed equally to this work.

Received: 21 December 2015 Accepted in revised form:

11 February 2016

Published online:

8 April 2016

\section{Introduction}

Central serous chorioretinopathy (CSC) is an acquired idiopathic disorder characterized by exudative detachment of the neurosensory retina and/or retinal pigment epithelium (RPE). It is more common in males than females, ${ }^{1,2}$ with an estimated incidence of 9.9 cases per 100000 men and 1.7 cases per 100000 women annually. ${ }^{3}$
E Moisseiev ${ }^{1}$, AJ Holmes ${ }^{1}$, A Moshiri and LS Morse

Acute CSC is usually self-limited, with recovery of visual acuity (VA), and absorption of subretinal fluid (SRF) within a few months, and patients are generally observed for 3 months allowing for spontaneous resolution. ${ }^{1,4,5}$ Use of glucocorticoids has been repeatedly found to be a risk factor for CSC, and their discontinuation is recommended when this diagnosis is made. ${ }^{1,2,6,7}$ When SRF persists and VA deteriorates, treatment is considered, most commonly with verteporfin photodynamic therapy (PDT), which is the most extensively studied treatment modality for CSC. $1,8-10$

The pathophysiology of CSC has not been fully elucidated. The most common theory of pathogenesis suggests hyperpermeability and increased hydrostatic pressure in the choroidal vasculature, which creates retinal pigment epithelial detachments and overwhelms the RPE barrier function leading to SRF accumulation. ${ }^{1,5,8,11-14}$ Because of the known association with glucocorticoids, it has been proposed that they cause CSC by potentiating vascular reactivity, ${ }^{15}$ inhibiting collagen synthesis in Bruch's membrane, ${ }^{16}$ or altering RPE function. ${ }^{17,18}$ As elevated levels of serum cortisol have been reported in patients with CSC, ${ }^{17,19}$ several studies have evaluated anti-glucocorticosteroid agents for its treatment, including ketoconazole, ${ }^{20,21}$ mifepristone, ${ }^{22,23}$ and rifampin. ${ }^{24-26}$

Elevated testosterone levels have also been implicated in the pathogenesis of CSC, in both men and women. ${ }^{17,27-29}$ Finasteride is an antiandrogenic agent that inhibits $5 \alpha$-reductase, the enzyme that converts testosterone to dihydrotestosterone, which binds with even greater affinity to the androgen receptor, ${ }^{30}$ and may therefore be a potentially effective treatment option for CSC. Only one small study investigated the use of finasteride in patients with CSC. ${ }^{31}$ The purpose of this study is to evaluate the safety and efficacy of finasteride treatment in patients with CSC. 


\section{Subjects and methods}

\section{Patient selection}

This is a retrospective chart review of all patients identified with CSC who were treated with finasteride at the University of California Davis Eye Center and Health System between 1 January 2011 and 31 December 2014. The electronic and archived paper medical records of all identified subjects were retrospectively reviewed. The protocol was approved by the University of California Davis Office of Human Research.

All patients had been diagnosed with CSC by a retina specialist based on the findings on clinical examination and characteristic findings on fluorescein angiography and/or optical coherence tomography (OCT)). All patients had documented presence of SRF at the time of finasteride initiation, and had symptoms or SRF for at least 3 months before treatment. Patients with any additional ocular pathology that could affect the retinal architecture and VA were excluded (such as diabetic retinopathy, age-related macular degeneration, optic neuropathy, advanced glaucoma, amblyopia, etc.). Patients with a history of ocular trauma or any ocular surgery other than uncomplicated cataract extraction were also excluded. All patients were 18 years or older. Women who were pregnant or breastfeeding were not offered this medicine, and all included female patients were not women of childbearing age. Patients whose records were incomplete or whose follow-up was $<3$ months were also excluded. In patients with bilateral involvement, both eyes were included if each fulfilled the inclusion criteria.

\section{Finasteride treatment}

All patients were treated with $5 \mathrm{mg}$ /day of finasteride orally. We used the $5 \mathrm{mg}$ /day dose as it is commercially available and was used in the only previous study evaluating finasteride in CSC. ${ }^{31}$ Treatment was administered for a minimum of 3 months, and was discontinued when SRF resolved. Treatment was also generally discontinued after 6 months even if SRF had not completely resolved, except for a few instances where the patients preferred to use it longer. All patients were seen 1 and 3 months after finasteride initiation. Given the drug's excellent safety profile, no additional laboratory tests were required for monitoring during the treatment period. At every visit they were asked whether they were taking the finasteride at their follow-up visits, to ensure compliance.

\section{Data collection and analysis}

The clinical data were retrieved from the medical records of included patients. Recorded parameters included patient demographics, previous medical and ocular history, history of steroid use, previous treatments for $\mathrm{CSC}$, length of finasteride treatment, additional treatments for CSC, VA, and intraocular pressure throughout the follow-up period, the occurrence of any complications, and OCT data. Data were collected at the time of finasteride initiation and throughout the followup at the final available visit. Specific time points included baseline at the time of finasteride initiation, after 1 month, 3 months, at the last time point under finasteride treatment, and at the final visit.

\section{Optical coherence tomography}

OCT scans were obtained by a spectral-domain instrument (Cirrus, Carl Zeiss Meditec AG, Jena, Germany). At each time point, the OCT scans were evaluated for the presence of SRF, and central macular thickness (CMT) values were recorded.

For the purpose of this study, treatment success was defined as resolution of SRF by OCT.

\section{Statistical analysis}

For statistical analysis all VA values were converted to the $\log$ MAR scale. According to Holladay $(X)$ and the University of Freiburg study group results $(X)$, blindness was set at 0.00125/2.9 (decimal/logMAR), light perception at $0.0025 / 2.6$, hand movements at $0.005 / 2.3$, and counting fingers at $0.014 / 1.85$. A paired two-tailed Student's $t$-test was used to compare means of continuous parameters between time points, and a $X^{2}$ test was used to analyse proportions of categorical parameters. Data were analysed using SPSS for windows version 17 (Chicago, IL, USA). A $P$-value of $<0.05$ was used to declare a statistically significant difference between groups.

\section{Results}

This series includes 29 eyes of 23 patients who met all of the inclusion criteria. There were 20 males $(86.9 \%)$ and 3 females (13.1\%), with a mean age of $53.2 \pm 14.1$ years (range $24-75$ years) at initiation of finasteride treatment. Ten $(43.4 \%)$ patients reported some kind of steroid use before developing CSC, including systemic treatment, inhalers, nasal sprays, and articular injections. Four of the 6 patients with bilateral CSC included in this study belonged to this group. Six (20.7\%) eyes underwent previous treatment for CSC before finasteride initiation (three had PDT, two received intravitreal anti-VEGF injections, and one was treated by both).

Mean duration of follow-up was $14.7 \pm 11.4$ months (range 3-42 months). Mean duration of finasteride 
treatment was 5.1 \pm 3.8 months (range 3-19 months). Finasteride treatment was given for 3 months in 16 (55.1\%) eyes, 4 months in 4 (13.8\%) eyes and 6 months in 5 (17.3\%) eyes. The remaining four $(13.8 \%)$ eyes belonged to four patients who continued treatment for over 6 months at their request. We note that for nine (31.0\%) eyes the last visit on finasteride treatment was also the last follow-up visit. These included four eyes of four patients who elected to continue the finasteride treatment beyond 6 months, three eyes of three patients who took finasteride for 3 months and had resolution of SRF, and it was discontinued, and two eyes of two patients who were treated for 3 months and were planned to continue for another 3 months. Only one (3.4\%) eye was pseudophakic at the time of finasteride initiation, and only one (3.4\%) more eye underwent cataract extraction during the follow-up period.

\section{Analysis of VA}

Mean initial VA at the time of finasteride initiation was $0.29 \pm 0.31 \log \mathrm{MAR}$ (equivalent to 20/40; range 20/20-20/400). One month following finasteride initiation, VA remained unchanged with a mean of $0.31 \pm 0.38 \log$ MAR $(P=0.80)$. However, a trend towards improved VA was noted at 3 months, with a mean of $0.25 \pm 0.36 \log$ MAR (equivalent to $20 / 36 ; P=0.07$ ). At 6 months mean VA was $0.28 \pm 34 \log \mathrm{MAR}(P=0.19)$, but only $11(37.9 \%)$ of eyes were still treated with finasteride at that time point. Mean VA at the last visit on finasteride was significantly improved to $0.22 \pm 0.28 \log \mathrm{MAR}$ (equivalent to $20 / 33 ; P=0.024$ ). A significant improvement in mean VA to $0.23 \pm 0.27 \log \mathrm{MAR}$ (equivalent to $20 / 34$; $P=0.026)$ was also found at the final available follow-up visit. These results are presented in Figure 1a.

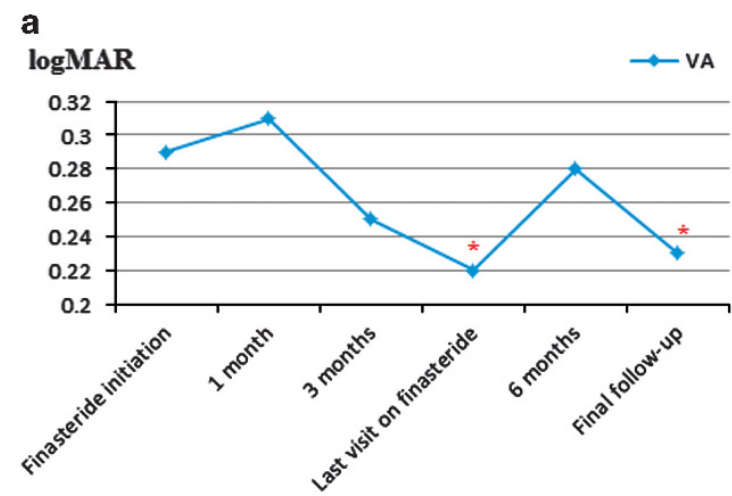

\section{Analysis of OCT data}

Mean CMT at time of finasteride initiation was $354 \pm 160 \mu \mathrm{m}$ (range $200-987 \mu \mathrm{m}$ ). This parameter was significantly reduced to $284 \pm 77 \mu \mathrm{m}(P=0.002)$ by 1 month. This effect was maintained throughout the follow-up period, with significantly reduced mean CMT values recorded after 3 months $(250 \pm 82 \mu \mathrm{m})$, after 6 months $(267 \pm 52 \mu \mathrm{m})$, at the last time point under finasteride treatment $(248 \pm 87 \mu \mathrm{m})$ and at the last visit $(247 \pm 85 \mu \mathrm{m} ; P=0.008,0.007,0.001$, and 0.001 , respectively). These results are presented in Figure $1 \mathrm{~b}$.

At the time of finasteride initiation, all eyes had SRF documented by OCT. The rate of SRF presence was significantly reduced from 100 to $66.7 \%$ at 1 month, $47.3 \%$ at 3 months, and $24.1 \%$ at the last visit under finasteride treatment $(P=0.0008,<0.0001$, and $<0.0001$, respectively). As resolution of SRF was defined as treatment success, these findings translate into success rates of $33.3 \%$ at 1 month, $52.7 \%$ at 3 months, and $75.9 \%$ at the final visit under finasteride treatment. All eyes included in this study were under finasteride treatment at these three time points. This success rate was maintained long term, as $21(72.4 \%)$ eyes had no SRF at the final follow-up $(P<0.0001)$.

An example of a patient who responded to finasteride treatment is provided in Figure 2.

\section{Complications, recurrences, and additional interventions}

No patient suffered any adverse effects that warranted discontinuation of finasteride. Additional surgery was performed in only one (3.4\%) eye during the follow-up period, and included uncomplicated cataract extraction that was unrelated to finasteride therapy.

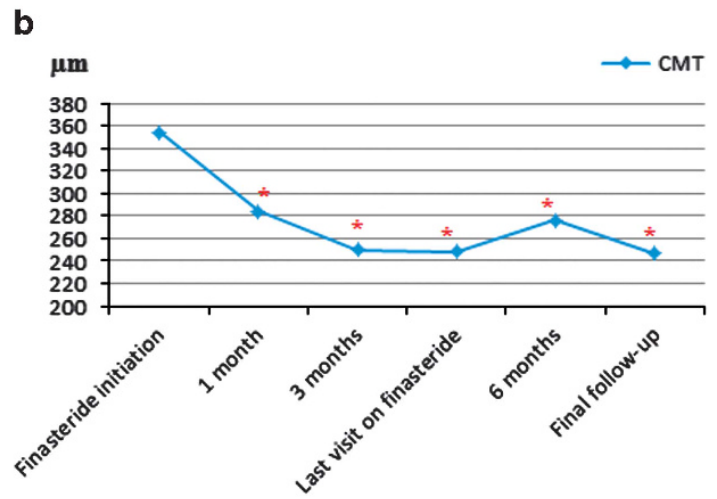

Figure 1 The effect of finasteride treatment on VA and CMT throughout the follow-up. A trend towards improved VA was noted after 3 months, but was lost at 6 months as only $37.9 \%$ eyes were still treated with it at that time. A statistically significant improvement in VA was found at the last visit on finasteride and at the final visit (a). CMT was significantly reduced at all time points compared with baseline (b). *indicates statistical significance. 
a

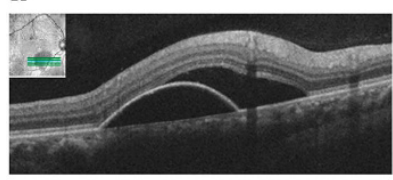

e

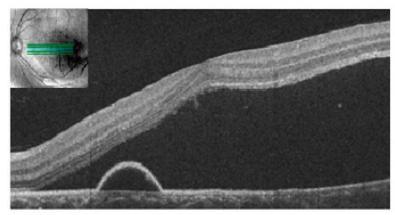

b

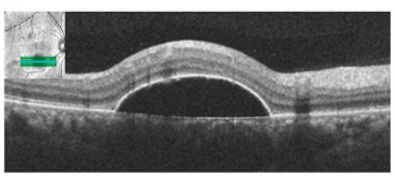

f

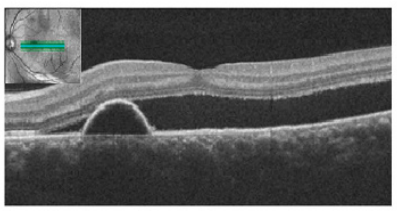

c

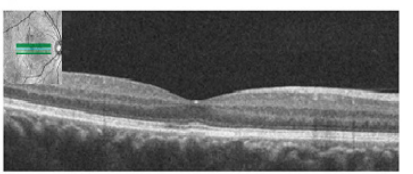

g

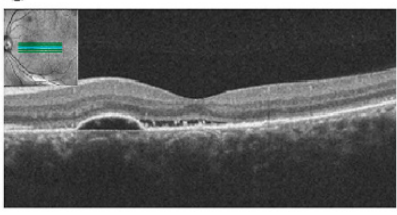

d

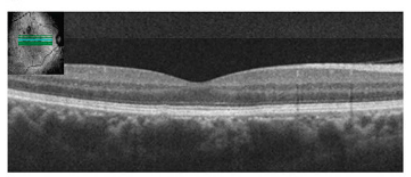

h

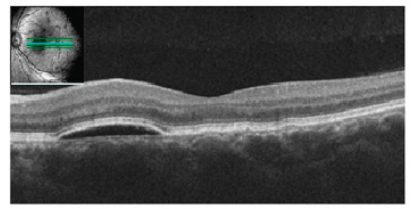

Figure 2 A 47-year-old male patient with no previous treatment who responded to finasteride treatment. He was treated for 6 months and did not require any additional treatments. Top row: the patient's right eye had non-foveal CSC, with a PED and SRF at presentation (a). The SRF was absorbed by 1 month (b), and the PED was virtually gone by 3 months (c). Normal foveal contour was documented at 3 months (c) and as long as 26 months after finasteride initiation (d). VA was 20/20 at all time points as the fovea was not involved. Bottom row: the patient's left eye presented with a PED and a significant amount of SRF, which included the fovea, and VA was reduced to 20/40 (e). By 1 month, a significant reduction in SRF was noted (f), and at 3 months only a trace amount of it was left, the PED decreased in size and VA improved to 20/30 (g). At the final follow-up (26 months after initiation of finasteride and 20 months after its discontinuation), a relatively flat PED remained with no SRF, and final VA was 20/25 (h).

Twenty-one (72.4\%) eyes had available follow-up after discontinuation of finasteride. Sixteen $(76.2 \%)$ of theses eyes were successfully treated with complete resolution of SRF at the time of finasteride discontinuation. Following discontinuation, recurrence of SRF was noted in six $(37.5 \%)$ of these eyes. Recurrence was noted after a mean of $3.7 \pm 1.3$ months (range $2-6$ months).

Seven $(24.1 \%)$ eyes in this series underwent PDT after finasteride treatment. Three of those were eyes that were treated successfully with finasteride but had recurrence of SRF after its discontinuation. The other three eyes that had recurrences were not treated by PDT as the amount of SRF was minimal or non-foveal, and were observed. Another four eyes were treated with PDT due to unsatisfactory response to finasteride. Of note is that three of the seven eyes that were treated with PDT also had previous treatment with PDT or intravitreal bevacizumab injections before initiation of finasteride treatment.

\section{Female patients}

This study included four eyes of three female patients, with a mean age of $61.0 \pm 1.6$ years. Under finasteride treatment, VA did not change significantly $(P=0.67)$, and only a modest reduction in mean CMT was noted, from $278 \pm 58 \mu \mathrm{m}$ to $244 \pm 49 \mu \mathrm{m}(P=0.44)$. However, SRF had resolved in two $(50 \%)$ of these eyes.

\section{Discussion}

The current mainstay of treatment for CSC is PDT, which has been demonstrated to be effective in resolving as well as preventing recurrences of this disorder. ${ }^{1,8-10}$ A recent large-scale collaboration study by the Macula Society reported a SRF resolution rate of $81 \%$ with PDT in patients with CSC. ${ }^{32}$ Nevertheless, PDT carries a potential risk for choroidal ischaemia, RPE atrophy, RPE rip, iatrogenic choroidal neovascularization, and vision loss, even with the generally preferred half-fluence settings. ${ }^{1,32-37}$ It has been shown that vascular endothelial growth factor (VEGF) levels are not elevated in patients with CSC, ${ }^{38,39}$ and use of intravitreal anti-VEGF agents has been found inferior to PDT. ${ }^{40}$ Therefore, these agents have a relatively minor and controversial role in the treatment of CSC. $1,41,42$

Oral treatment for CSC is an appealing idea, if it can be proven to be effective and systemically safe. Such a treatment could be the first treatment choice for patients with CSC who require intervention, to which PDT could be added later on if necessary. It should be noted that the $75.9 \%$ rate of SRF resolution achieved in this series is comparable to that reported with PDT $\left(81 \%{ }^{32}\right)$. Because of the known association with glucocorticosteroids, the most widely studied oral agents for the treatment of CSC are anti-glucocorticoids, such as ketoconazole, ${ }^{20,21}$ mifepristone, ${ }^{22,23}$ and rifampin. ${ }^{24-26}$ Other reports investigated anti-mineralocorticoids, such as eplerenone $e^{43-46}$ and spironolactone, ${ }^{47,48}$ as well as carbonic anhydrase inhibitors (acetazolamide). ${ }^{49}$ We note that all these series were small (under 20 patients) with relatively short follow-up, and reported mixed results. A reduction in CMT and SRF was noted with the three anti-glucocorticoids, although no change in VA was achieved with ketoconazole and mifepristone. ${ }^{20-23}$ The largest series of CSC patients treated with rifampin reported significant improvement in VA, but two of the 
14 patients had to discontinue the medicine owing to systemic adverse events. ${ }^{26}$ It should also be noted that one patient treated with rifampin for CSC was reported to develop hepatotoxicity. ${ }^{50}$ Reduction of CMT and improvement in VA was reported with the antimineralocorticoids, but it should be noted that these agents can cause low blood pressure, hyperkalemia, and other adverse effects. ${ }^{43-48}$ Acetazolamide was shown to reduce CMT but had no effect on VA, and it too can cause low blood pressure and other adverse effects. ${ }^{49}$

Finasteride is approved by the FDA for the treatment of benign prostatic hyperplasia (BPH) and androgenic alopecia, and is known to have an excellent safety profile, ${ }^{51-53}$ and has also been shown to have fewer side effects and drug interactions than ketoconazole. ${ }^{54}$ Its most common adverse effect is decreased libido, and no laboratory workup is required except for monitoring of prostate-specific antigen in men with $\mathrm{BPH}$ who are using it long-term. In the only previous study of its use in CSC, finasteride was shown to improve CMT and SRF in five patients who were treated with it for 3 months and followed for 3 more, with no adverse events. ${ }^{31}$ Our results support the hypothesis that finasteride may be an effective treatment for CSC. VA was not improved in the previous study; ${ }^{31}$ however, our results revealed a trend towards improved VA starting after 3 months of finasteride treatment, which reached statistical significance at the end of the treatment course. In addition, a significant reduction in CMT and absorption of SRF was found at all time points, starting 1 month after finasteride initiation and lasting until the end of the follow-up period. No adverse events were noted in any patient, over a mean follow-up period of 16.1 months. Overall, treatment with finasteride achieved complete resolution of SRF in $75.9 \%$ of eyes, and the rate of SRF recurrence after its discontinuation was $37.5 \%$, with all noted recurrences occurring within 6 months. Another important aspect in which finasteride may offer an advantage is the financial one. Although prices may vary, at the US the cost of 1 month's supply of $5 \mathrm{mg}$ finasteride pills is generally $<50 \$$, considerably less than the cost of a single PDT session.

Our series also includes the first report of finasteride use for the treatment of CSC in female patients, as the previous report included only five males. ${ }^{31}$ The majority of our series included male patients, as CSC is more common in males, and finasteride was not given to female patients with childbearing potential. However, we were able to include four eyes of three female patients with a mean age of $64.8 \pm 8.6$ years. Under finasteride treatment, VA and CMT did not change significantly, but this is expected given the small number of cases in this subgroup. However, SRF had completely resolved in two $(50 \%)$ eyes of two of these patients. We note that similar results that did not reach statistical significance were reported for the previous study in five male patients. ${ }^{31}$ Therefore, we believe our results may indicate some clinical usefulness of finasteride in female patients as well.

This is a pilot study with relatively short follow-up. Additional limitations of this study include its retrospective nature, lack of monthly follow-up, and its sample size. However, we note that this is the largest series of CSC patients treated with an oral anti-hormonal agent, and it was large enough to achieve statistically significant results. Another limitation is the inclusion of six $(20.7 \%)$ eyes that had previous treatment for CSC before finasteride, and eight $(24.1 \%)$ that underwent subsequent PDT. We note that three eyes belonged to both of these groups, and may represent more severe and refractory cases. In addition, we note that the initial mean VA was relatively good (0.30 logMAR; equivalent to $20 / 40$ Snellen) and that nine (30\%) eyes had non-foveal involvement. These factors may have caused a ceiling effect preventing the visual improvement from achieving stronger statistical significance. Finally, we note that CSC has a high rate of spontaneous resolution, and as the study does not include an observational control group, it is possible that spontaneous resolution is responsible for some of the improvement in VA and SRF resolution that was noted with finasteride treatment.

In conclusion, our results strengthen the findings previously reported, ${ }^{31}$ and support the role of androgens in the pathogenesis of CSC and of finasteride for its treatment, even in female patients. Owing to its remarkable effect in reducing CMT and SRF, and excellent safety profile, we suggest it may be tried in the initial treatment of patients with CSC. Finasteride was found to have an impressive rate of success in SRF resolution, and a lower rate of recurrence after its discontinuation. We recommend a trial of finasteride for 3-6 months in patients with CSC: if treatment is successful, patients should be followed for recurrences especially during the first 6 months following finasteride discontinuation. Cases with recurrence of SRF can be observed, re-treated with finasteride or referred for PDT according to their severity. It finasteride treatment is not successful, it should be stopped and PDT or alternative treatments considered. Patients who have SRF recurrence after discontinuing finasteride after initial success can be considered for long-term treatment with it, as data from patients chronically treated with it for other conditions indicate its safety. ${ }^{51-53}$ We note that four patients in our series used finasteride for longer than 6 months with no adverse effects. Further large-scale randomized controlled studies of the dosage and duration of finasteride treatment in patients with CSC will be needed to further validate our findings and establish guidelines for its optimal use. 


\section{Summary}

\section{What was known before}

- The pathophysiology of central serous chorioretinopathy (CSC) is complex and not fully elucidated. Glucocorticoids are known to be involved, and elevated testosterone levels have also been implicated in it.

- Several studies have investigated oral treatments for CSC, but these have focused mainly on anti-glucocorticoid agents.

- Only one small study (5 patients) has previously investigated finasteride, an anti-androgenic agent, for the treatment of CSC.

\section{What this study adds}

- This is only the second study to investigate finasteride for the treatment of CSC.

- This is the largest series (29 eyes), and provides more information on the effects of finasteride on patients with CSC. Of note is that this is also the largest series of CSC patients treated with an oral anti-hormonal agent (not only finasteride).

- We report an excellent safety profile, as well as impressive efficacy regarding improvement in visual acuity and reduction, and resolution in subretinal fluid.

- This is also the first report of the efficacy of finasteride in female patients with CSC.

- The results of this study may influence the way CSC patients are managed.

\section{Conflict of interest}

The authors declare no conflict of interest.

\section{References}

1 Nicholson B, Noble J, Forooghian F, Meyerle C. Central serous chorioretinopathy: update on pathophysiology and treatment. Surv Ophthalmol 2013; 58: 103-126.

2 Tittl MK, Spaide RF, Wong D, Pilotto E, Yannuzzi LA, Fisher YL et al. Systemic findings associated with central serous chorioretinopathy. Am J Ophthalmol 1999; 128: 63-68.

3 Kitzmann AS, Pulido JS, Diehl NN, Hodge DO, Burke JP. The incidence of central serous chorioretinopathy in Olmsted County, Minnesota, 1980-2002. Ophthalmology 2008; 115: 169-173.

4 Klein ML, Van Buskirk EM, Friedman E, Gragoudas E, Chandra S. Experience with nontreatment of central serous choroidopathy. Arch Ophthalmol 1974; 91: 247-250.

5 Liegl R, Ulbig MW. Central serous chorioretinopathy. Ophthalmologica 2014; 232: 65-76.

6 Wakakura M, Ishikawa S. Central serous chorioretinopathy complicating systemic corticosteroid treatment. $\mathrm{Br} \mathrm{J}$ Ophthalmol 1984; 68: 329-331.

7 Levy J, Marcus M, Belfair N, Klemperer I, Lifshitz T. Central serous chorioretinopathy in patients receiving systemic corticosteroid therapy. Can J Ophthalmol 2005; 40: 217-221.

8 Erikitola OC, Crosby-Nwaobi R, Lotery AJ, Sivaprasad S. Photodynamic therapy for central serous chorioretinopathy. Eye (Lond) 2014; 28: 944-957.
9 Yannuzzi LA, Slakter JS, Gross NE, Spaide RF, Costa D, Huang SJ et al. Indocyanine green angiography-guided photodynamic therapy for treatment of chronic central serous chorioretinopathy: a pilot study. Retina 2003; 23: 288-298.

10 Wu ZH, Lai RY, Yip YW, Chan WM, Lam DS, Lai TY. Improvement in multifocal electroretinography after halfdose verteporfin photodynamic therapy for central serous chorioretinopathy: a randomized placebo-controlled trial. Retina 2011; 31: 1378-1386.

11 Prünte C, Flammer J. Choroidal capillary and venous congestion in central serous chorioretinopathy. $A m \mathrm{~J}$ Ophthalmol 1996; 121: 26-34.

12 Spaide RF, Hall L, Haas A, Campeas L, Yannuzzi LA, Fisher YL et al. Indocyanine green videoangiography of older patients with central serous chorioretinopathy. Retina 1996; 16: 203-213.

13 Iida T, Kishi S, Hagimura N, Shimizu K. Persistent and bilateral choroidal vascular abnormalities in central serous chorioretinopathy. Retina 1999; 19: 508-512, 513.

14 Giovannini A, Scassellati-Sforzolini B, D'Altobrando E, Mariotti C, Rutili T, Tittarelli R. Choroidal findings in the course of idiopathic serous pigment epithelium detachment detected by indocyanine green videoangiography. Retina 1997; 17: 286-293.

15 Yang S, Zhang L. Glucocorticoids and vascular reactivity. Curr Vasc Pharmacol 2004; 2: 1-12.

16 Oikarinen AI, Uitto J, Oikarinen J. Glucocorticoid action on connective tissue: from molecular mechanisms to clinical practice. Med Biol 1986; 64: 221-230.

17 Haimovici R, Rumelt S, Melby J. Endocrine abnormalities in patients with central serous chorioretinopathy. Ophthalmology 2003; 110: 698-703.

18 Arndt C, Sari A, Ferre M, Parrat E, Courtas D, De Seze J et al. Electrophysiological effects of corticosteroids on the retinal pigment epithelium. Invest Ophthalmol Vis Sci 2001; 42: 472-475.

19 Garg SP, Dada T, Talwar D, Biswas NR. Endogenous cortisol profile in patients with central serous chorioretinopathy. Br J Ophthalmol 1997; 81: 962-964.

20 Golshahi A, Klingmüller D, Holz FG, Eter N. Ketoconazole in the treatment of central serous chorioretinopathy: a pilot study. Acta Ophthalmol 2010; 88: 576-581.

21 Meyerle CB, Freund KB, Bhatnagar P, Shah V, Yannuzzi LA. Ketoconazole in the treatment of chronic idiopathic central serous chorioretinopathy. Retina 2007; 27: 943-946.

22 Nielsen JS, Weinreb RN, Yannuzzi L, Jampol LM. Mifepristone treatment of chronic central serous chorioretinopathy. Retina 2007; 27: 119-122.

23 Nielsen JS, Jampol LM. Oral mifepristone for chronic central serous chorioretinopathy. Retina 2011; 31: 1928-1936.

24 Steinle NC, Gupta N, Yuan A, Singh RP. Oral rifampin utilisation for the treatment of chronic multifocal central serous retinopathy. Br J Ophthalmol 2012; 96: 10-13.

25 Pouw AE, Olmos de Koo LC. Oral rifampin for central serous retinopathy: a strategic approach in three patients. Ophthalmic Surg Lasers Imaging Retina 2015; 46: 98-102.

26 Shulman S, Goldenberg D, Schwartz R, Habot-Wilner Z, Barak A, Ehrlich $\mathrm{N}$ et al. Oral Rifampin treatment for longstanding chronic central serous chorioretinopathy. Graefes Arch Clin Exp Ophthalmol 2015; 254: 15-22.

27 Nudleman E, Witmer MT, Kiss S, Williams GA, Wolfe JD. Central serous chorioretinopathy in patients receiving exogenous testosterone therapy. Retina 2014; 34: 2128-2132. 
28 Grieshaber MC, Staub JJ, Flammer J. The potential role of testosterone in central serous chorioretinopathy. $\mathrm{Br} \mathrm{J}$ Ophthalmol 2007; 91: 118-119.

29 Ahad MA, Chua CN, Evans NM. Central serous chorioretinopathy associated with testosterone therapy. Eye (Lond) 2006; 20: 503-505.

30 Grino PB, Griffin JE, Wilson JD. Testosterone at high concentrations interacts with the human androgen receptor similarly to dihydrotestosterone. Endocrinology 1990; 126: 1165-1172.

31 Forooghian F, Meleth AD, Cukras C, Chew EY, Wong WT, Meyerle CB. Finasteride for chronic central serous chorioretinopathy. Retina 2011; 31: 766-771.

32 Lim JI, Glassman AR, Aiello LP, Chakravarthy U, Flaxel CJ, Spaide RFMacula Society CSC Collaborative Study GroupResearch and Education Committee and Website Committee. Collaborative retrospective macula society study of photodynamic therapy for chronic central serous chorioretinopathy. Ophthalmology 2014; 121: 1073-1078.

33 Lai TY, Chan WM, Li H, Lai RY, Liu DT, Lam DS. Safety enhanced photodynamic therapy with half dose verteporfin for chronic central serous chorioretinopathy: a short term pilot study. Br J Ophthalmol 2006; 90: 869-874.

34 Reibaldi M, Boscia F, Avitabile T, Russo A, Cannemi V, Uva MG et al. Low-fluence photodynamic therapy in longstanding chronic central serous chorioretinopathy with foveal and gravitational atrophy. Eur J Ophthalmol 2009; 19: 154-158.

35 Reibaldi M, Cardascia N, Longo A, Furino C, Avitabile T, Faro $\mathrm{S}$ et al. Standard-fluence versus low-fluence photodynamic therapy in chronic central serous chorioretinopathy: a nonrandomized clinical trial. Am J Ophthalmol 2010; 149: 307-315.

36 Williams MA, Mulholland C, Silvestri G. Photodynamic therapy for central serous chorioretinopathy using a reduced dose of verteporfin. Can J Ophthalmol 2008; 43: 123.

37 Koytak A, Erol K, Coskun E, Asik N, Öztürk H, Özertürk Y. Fluorescein angiography-guided photodynamic therapy with half-dose verteporfin for chronic central serous chorioretinopathy. Retina 2010; 30: 1698-1703.

38 Lim JW, Kim MU, Shin MC. Aqueous humor and plasma levels of vascular endothelial growth factor and interleukin-8 in patients with central serous chorioretinopathy. Retina 2010; 30: 1465-1471.

39 Shin MC, Lim JW. Concentration of cytokines in the aqueous humor of patients with central serous chorioretinopathy. Retina 2011; 31: 1937-1943.

40 Bae SH, Heo J, Kim C, Kim TW, Shin JY, Lee JY et al. Low-fluence photodynamic therapy versus ranibizumab for chronic central serous chorioretinopathy: one-year results of a randomized trial. Ophthalmology 2014; 121: 558-565.

41 Park SU, Lee SJ, Kim M. Intravitreal anti-vascular endothelial growth factor versus observation in acute central serous chorioretinopathy: one-year results. Korean I Ophthalmol 2014; 28: 306-313.

42 Chung YR, Seo EJ, Lew HM, Lee KH. Lack of positive effect of intravitreal bevacizumab in central serous chorioretinopathy: meta-analysis and review. Eye (Lond) 2013; 27: 1339-1346.

43 Salz DA, Pitcher JD 3rd, Hsu J, Regillo CD, Fineman MS, Elliott KS et al. Oral eplerenone for treatment of chronic central serous chorioretinopathy: a case series. Ophthalmic Surg Lasers Imaging Retina 2015; 46: 439-444.

44 Singh RP, Sears JE, Bedi R, Schachat AP, Ehlers JP, Kaiser PK. Oral eplerenone for the management of chronic central serous chorioretinopathy. Int J Ophthalmol 2015; 8: 310-314.

45 Breukink MB, den Hollander AI, Keunen JE, Boon CJ, Hoyng CB. The use of eplerenone in therapy-resistant chronic central serous chorioretinopathy. Acta Ophthalmol 2014; 92: e488-e490.

46 Bousquet E, Beydoun T, Zhao M, Hassan L, Offret O, Behar-Cohen F. Mineralocorticoid receptor antagonism in the treatment of chronic central serous chorioretinopathy: a pilot study. Retina 2013; 33: 2096-2102.

47 Bousquet E, Beydoun T, Rothschild PR, Bergin C, Zhao M, Batista $\mathrm{R}$ et al. Spironolactone for nonresolving central serous chorioretinopathy: a randomized controlled crossover study. Retina 2015; 35: 2505-2515.

48 Herold TR, Prause K, Wolf A, Mayer WJ, Ulbig MW. Spironolactone in the treatment of central serous chorioretinopathy - a case series. Graefes Arch Clin Exp Ophthalmol 2014; 252: 1985-1991.

49 Pikkel J, Beiran I, Ophir A, Miller B. Acetazolamide for central serous retinopathy. Ophthalmology 2002; 109: 1723-1725.

50 Nelson J, Saggau DD, Nielsen JS. Rifampin induced hepatotoxicity during treatment for chronic central serous chorioretinopathy. Retin Cases Brief Rep 2014; 8: 70-72.

51 Gubelin Harcha W, Barboza Martínez J, Tsai TF, Katsuoka K, Kawashima M, Tsuboi $\mathrm{R}$ et al. A randomized, active- and placebo-controlled study of the efficacy and safety of different doses of dutasteride versus placebo and finasteride in the treatment of male subjects with androgenetic alopecia. J Am Acad Dermatol 2014; 70: 489-498.

52 Belknap SM, Aslam I, Kiguradze T, Temps WH, Yarnold PR, Cashy $\mathrm{J}$ et al. Adverse event reporting in clinical trials of finasteride for androgenic alopecia: a meta-analysis. JAMA Dermatol 2015; 151: 600-606.

53 Gupta AK, Charrette A. The efficacy and safety of $5 \alpha$-reductase inhibitors in androgenetic alopecia: a network meta-analysis and benefit-risk assessment of finasteride and dutasteride. J Dermatolog Treat 2014; 25: 156-161.

54 Venturoli S, Marescalchi O, Colombo FM, Macrelli S, Ravaioli B, Bagnoli A et al. A prospective randomized trial comparing low dose flutamide, finasteride, ketoconazole, and cyproterone acetate-estrogen regimens in the treatment of hirsutism. J Clin Endocrinol Metab 1999; 84: 1304-1310. 